ARTICLE

Received 18 Sep 2014 | Accepted 17 Feb 2015 | Published 26 Mar 2015

\title{
The genome-wide effects of ionizing radiation on mutation induction in the mammalian germline
}

Adeolu B. Adewoye ${ }^{1, \star}$, Sarah J. Lindsay ${ }^{2, \star}$, Yuri E. Dubrova ${ }^{1} \&$ Matthew E. Hurles $^{2}$

The ability to predict the genetic consequences of human exposure to ionizing radiation has been a long-standing goal of human genetics in the past 50 years. Here we present the results of an unbiased, comprehensive genome-wide survey of the range of germline mutations induced in laboratory mice after parental exposure to ionizing radiation and show irradiation markedly alters the frequency and spectrum of de novo mutations. Here we show that the frequency of de novo copy number variants (CNVs) and insertion/deletion events (indels) is significantly elevated in offspring of exposed fathers. We also show that the spectrum of induced de novo single-nucleotide variants (SNVs) is strikingly different; with clustered mutations being significantly over-represented in the offspring of irradiated males. Our study highlights the specific classes of radiation-induced DNA lesions that evade repair and result in germline mutation and paves the way for similarly comprehensive characterizations of other germline mutagens.

\footnotetext{
${ }^{1}$ Department of Genetics, University of Leicester, Leicester LE1 7RH, UK. ${ }^{2}$ Wellcome Trust Sanger Institute, Wellcome Trust Genome Campus, Hinxton, Cambridge CB10 1SA, UK. * These authors contributed equally to this work. Correspondence and requests for materials should be addressed to Y.E.D. (email: yed2@le.ac.uk) or to M.E.H. (email: meh@sanger.ac.uk).
} 
onizing radiation (IR) is an extensively studied mutagenic agent, exposure to which results in different types of DNA damage, ranging from modified nucleotides to double-stand breaks ${ }^{1}$. The mutagenic effects of IR on the germline are of particular concern as they lead to the accumulation of extra mutations in the offspring of irradiated parents ${ }^{2}$. Despite numerous efforts ${ }^{3}$, little is known about the genetic effects of radiation exposure in humans and the only definitive evidence for germline mutation induction in vivo in mammals comes from mouse studies ${ }^{2,4}$. Moreover, the current estimates of the germline genetic hazards of radiation and other mutagens for humans have been derived from the results obtained from the analysis of only a handful of protein-coding genes (typically seven) ${ }^{4,5}$. Although the results of these studies provide important information regarding the pattern of mutation induction in the germline of irradiated parents, the analysis of just seven protein-coding genes cannot accurately predict the genome-wide pattern of mutation induction. The lack of genome-wide data describing the pattern of mutation induction by IR or other mutagens also precludes further extrapolation from the results of mouse studies on the predicted excess of genetic syndromes among the offspring of exposed parents. Recent advances in genetic technologies have provided new microarray-based and next-generation sequencingbased tools for the genome-wide analysis of genetic variation, which have the potential for characterizing germline mutation in humans and mice. Here we present the results of the first systematic study aimed to analyse the genome-wide effects of IR on mutation induction in the mouse germline.

\section{Results}

Experimental design. We carried out a matched case-control experiment to investigate the immediate and long-term effects of IR on germline mutation in mice. Fifteen C57BL6/J male mice

a

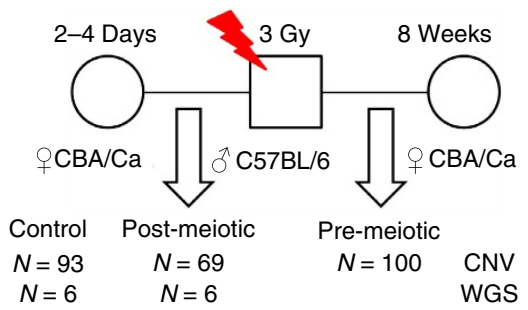

b
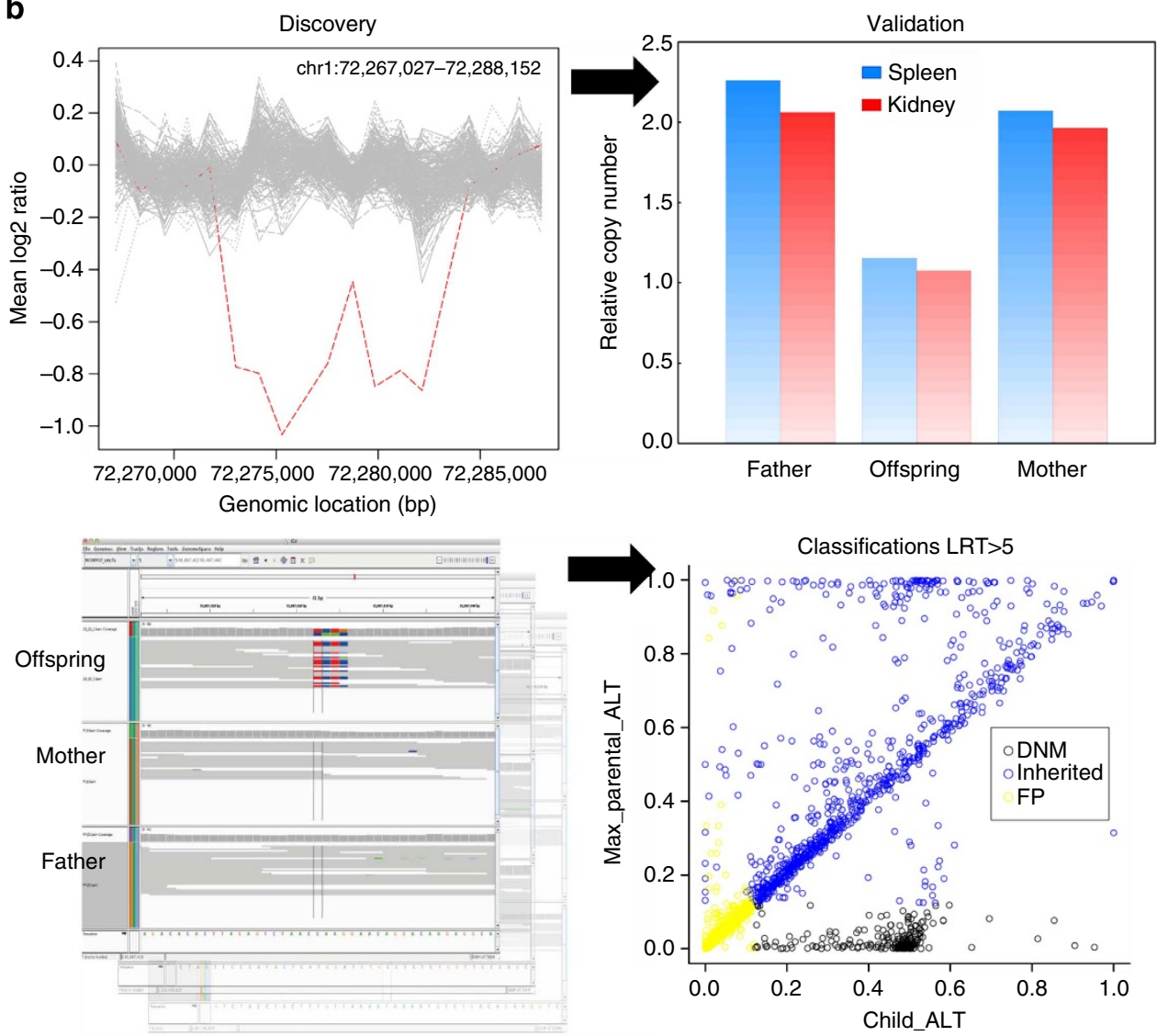

Figure 1 | Experimental design and discovery of de novo mutations. (a) Design of mutational study. The number of offspring analysed by comparative genome hybridization (CNV) and whole-genome sequencing (WGS) is shown. (b) Discovery and validation of de novo mutations. The panel depicts a representative CNV profile with a 9.1-kb deletion (left) validated by GPCR (right). The bottom panel displays a sequence alignment and discovery of a multisite variant in one offspring (left), and validation of all putative de novo SNVs and indels in all offspring (right), where variants are classified as true de novo variants, inherited, or false positive categories based on read count proportions of the alternative allele. 
were irradiated at 8-week old, and then mated with two different $\mathrm{CBA} / \mathrm{Ca}$ females 3 days and then 8 weeks post irradiation (Fig. 1a), thus enabling us to study mutation induction at postmeiotic and pre-meiotic stages of spermatogenesis, respectively ${ }^{4}$. To establish the rate of de novo genomic rearrangements resulting in copy number variation in the germline, we used microarraybased comparative genomic hybridization to compare the copy number of case and control offspring and their parents genomewide with a resolution of $\sim 5.5 \mathrm{~kb}$ (Methods). All de novo CNVs were validated by quantitative PCR (qPCR; Fig. 1b). In addition, we carried out whole-genome HiSeq sequencing $(>22 \mathrm{X}$ coverage) on four matched exposed and control pedigrees (Fig. 1a) from matings at the post-meiotic stage in order to capture induced de novo SNVs and indels $(<50 \mathrm{bp})$ in the offspring derived from un-repaired radiation-damaged sperm (Fig. 1b). To capture unusual or clustered mutations that may be under-ascertained using standard analytical pipelines, we used several different mutation callers and parameters to call de novo $S N V s$ and indels in control and exposed offspring (Methods). We minimized false positives in our analyses by focusing on the nonrepetitive portion of the mouse genome, leaving on average, $89.1 \%$ of the mappable genome for analysis. After discovery, putative de novo SNV and indel candidates were validated using longer read sequencing (Miseq) of PCR amplicons allowing definitive classification of $96 \%$ of candidate sites.

De novo CNV mutations. The frequency per offspring of de novo CNVs in control families is similar to that reported in the previous work ${ }^{6}$. It should be noted that in the offspring of irradiated males we also found four instances of germline mosaicism where the same de novo CNV mutation was detected in two or more offspring (Fig. 2). Given that recent exposure to IR during adulthood cannot result in accumulation of mosaic mutations in the paternal germline, these were regarded as spontaneous mutations and excluded from the estimates of mutation rate but used for the analysis of CNV spectra. Our data are in line with the results of recent study showing that mosaicism among parents of children with CNVs is quite common ${ }^{7}$.

We found a significant eightfold increase in the frequency of de novo $\mathrm{CNVs}$ in the offspring from exposed fathers conceived both pre-meiotically and post-meiotically compared with control offspring (Fig. 3a and Table 1). We could determine the parental origin for ten de novo CNVs in cases, with all arising in the paternal germline (Supplementary Table 1), demonstrating increased de novo $\mathrm{CNV}$ induction by paternal exposure to IR. Using the frequency of de novo CNVs found in the offspring of control and irradiated parents, we estimated the doubling dose for $\mathrm{CNV}$ mutation as $0.45 \mathrm{~Gy}(95 \% \mathrm{CI}=0-2.62 \mathrm{~Gy})$, a value close to those obtained in mice using traditional mutation scoring systems ${ }^{8}$. Among the 14 unique germline CNV mutations found in the offspring of irradiated males, 12 were deletions and other 2 were duplications (Supplementary Table 1). We found that the spectrum of radiation-induced CNVs is enriched for very large deletions $(>1,000 \mathrm{~kb})$ with the loss of up to 80 genes, mostly protein-coding (Supplementary Data 1 and Fig. 3b), which could potentially be deleterious (Fig. 3b).

De novo SNV and indel mutations. We found that the germline mutation rate for SNVs in mice is less than half that of humans ${ }^{9}$, with a mean across all control offspring of $3.75 \times 10^{-9}$ per nucleotide per generation. Similarly, the indel mutation rate in control offspring is also lower than in humans ${ }^{9}$, although the
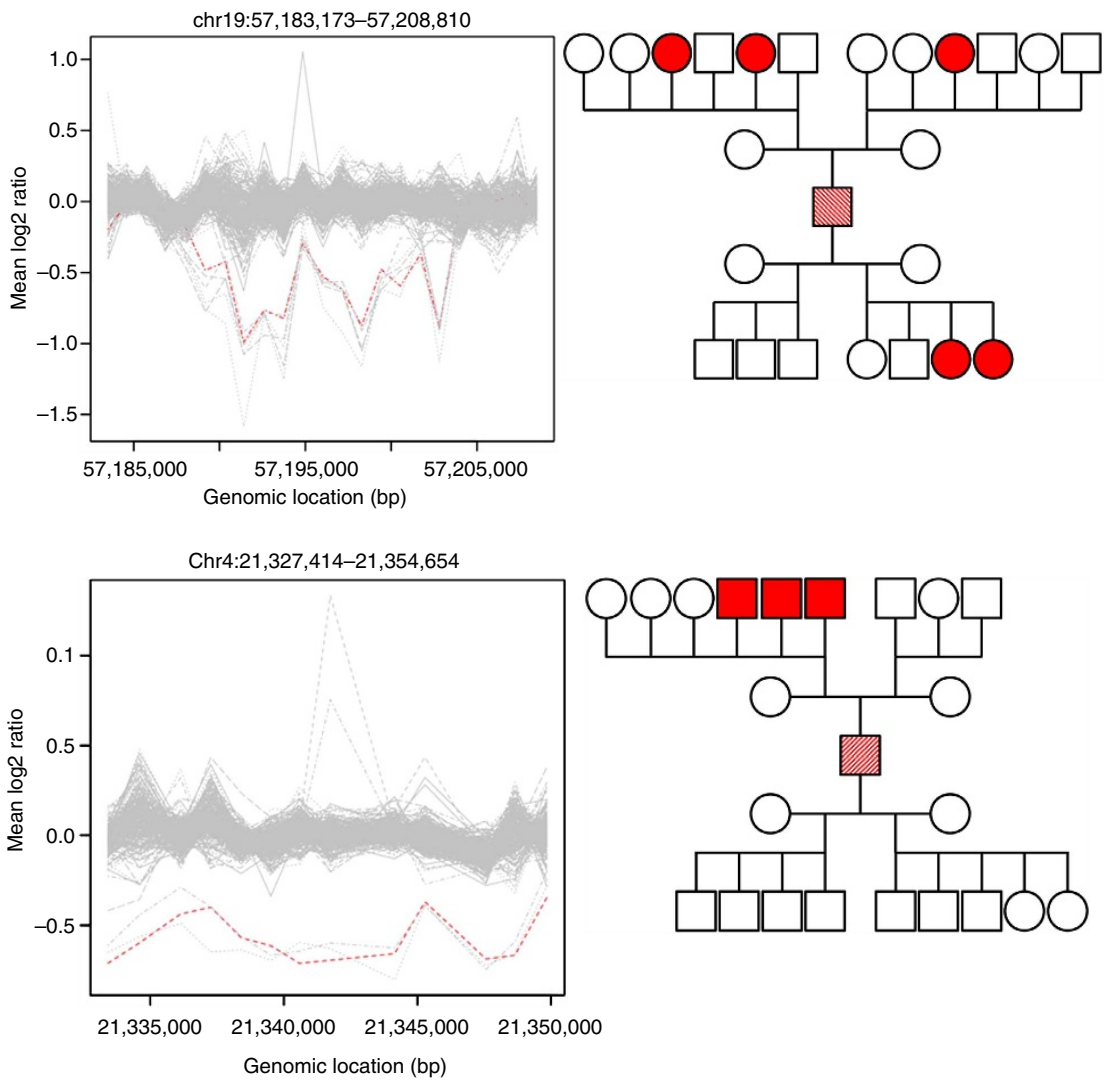

Figure 2 | Examples of mosaic de novo CNV mutations. Panel on the left represents CNV plots; panel on the right shows segregation of mosaic mutations (in red). The carriers of mosaic mutation are show in hatched red. 
a

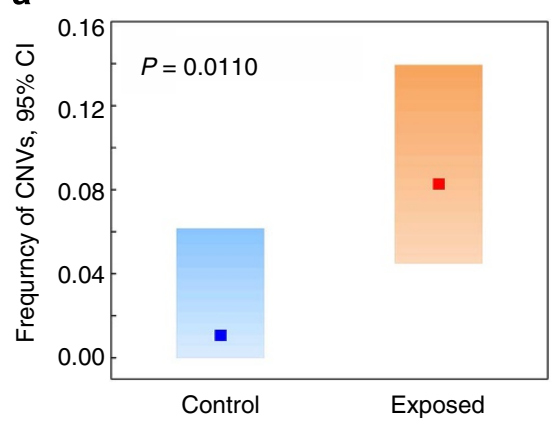

C

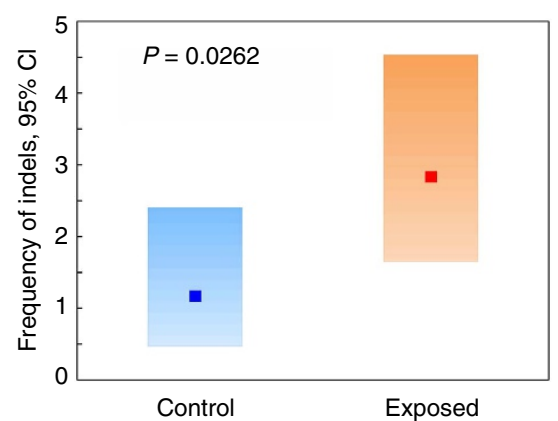

e

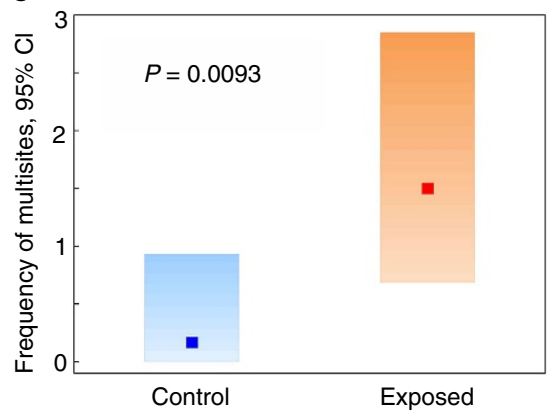

b

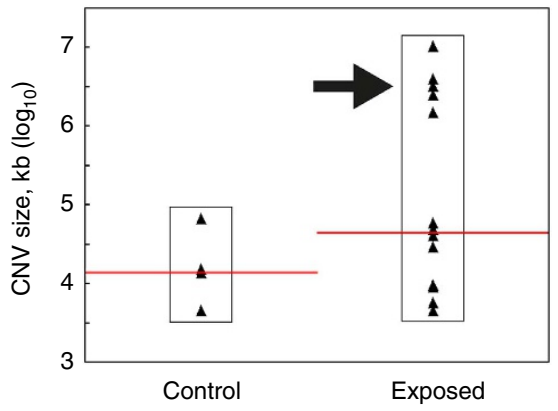

d

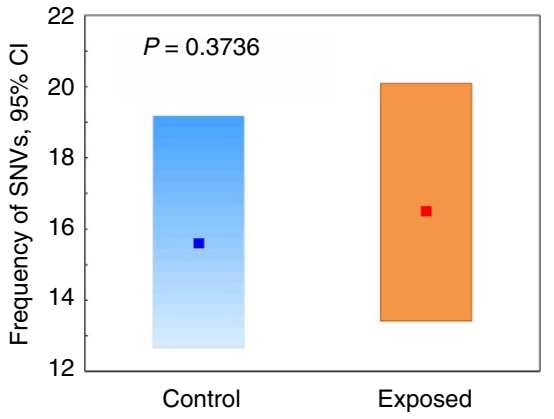

Figure 3 | De novo mutation frequencies and spectrum. (a) Frequency of de novo CNV mutations in the offspring of control and irradiated males. (b) Variability plot showing the spectrum of de novo CNV mutations in the offspring of control and irradiated males $(P=0.1533$, Kruskal-Wallis test). The median values are shown in red; a group of very large CNVs found in the offspring of irradiated males ( $>1,000 \mathrm{~kb}$ ) is arrowed. (c) Frequency of de novo indel mutations in the offspring of control and irradiated males. (d) Frequency of de novo SNV mutations in the offspring of control and irradiated males. (e) Frequency of de novo multisite mutations in the offspring of control and irradiated males. The frequency of de novo mutations per offspring and probability of difference from controls is shown on all graphs. For CNVs the probabilities were estimated using Fisher's exact test; for SNVs, indels and multisites, the probabilities were estimated using a one-tailed test based on Poisson simulation of mutations in exposed and control populations (Methods). $\mathrm{Cl}$, confidence interval.

small numbers preclude a more quantitative evaluation (Supplementary Table 2). Through interrogation of nearby informative heterozygous sites, we could ascertain the parental origin for $27 \mathrm{SNVs}$ in controls, and showed a ratio of 20:7 paternal/maternal origin, which is similar to the established ratio in humans ${ }^{10}$. The structure of shared haplotypes among different lines of inbred laboratory mice ${ }^{11}$ prohibits the phasing of many de novo SNVs.

We found that the rate of induction of de novo indels was significantly increased, by a factor of 2.4 , in offspring of irradiated males (Fig. 3c, Table 1), compared with controls. In contrast, the number of de novo SNVs in offspring of exposed fathers is not significantly elevated compared with control offspring (Fig. 3d, Table 1). Although the baseline rate of de novo SNVs in the germline of irradiated parents does not significantly exceed that in controls, the spectrum of mutation is markedly different. Specifically, we found the frequency of clustered de novo mutations (clusters of 1-4 SNVs or clusters of 1-2 SNVs and indels within a few base-pairs of each other, Fig. 4), to be significantly elevated in the offspring of irradiated fathers compared with controls (Fig. $3 e$ and Table 1). Although the presence of a single occurrence of clustered mutation in a control offspring supports the notion that clustered mutations occur at a low level in unexposed individuals ${ }^{12,13}$, the enrichment of clustered mutations in the offspring of exposed males can be attributed to the induction of clustered damaged sites in the germline of irradiated males. Clustered DNA-damaged sites, defined as two or more lesions within one or two helical turns of the DNA, are regarded as a signature of radiation exposure ${ }^{14,15}$. Judging from the results on the induction of clustered DNA damage sites in irradiated mammalian cells ${ }^{16}$, exposure to $3 \mathrm{~Gy}$ of X-rays may induce up to 1,000 multiply damaged sites in the germline of irradiated male mice. Given that the efficiency of their repair is substantially compromised and delayed ${ }^{15,16}$, 
Table 1 | De novo mutation frequencies in the offspring of control and irradiated parents.

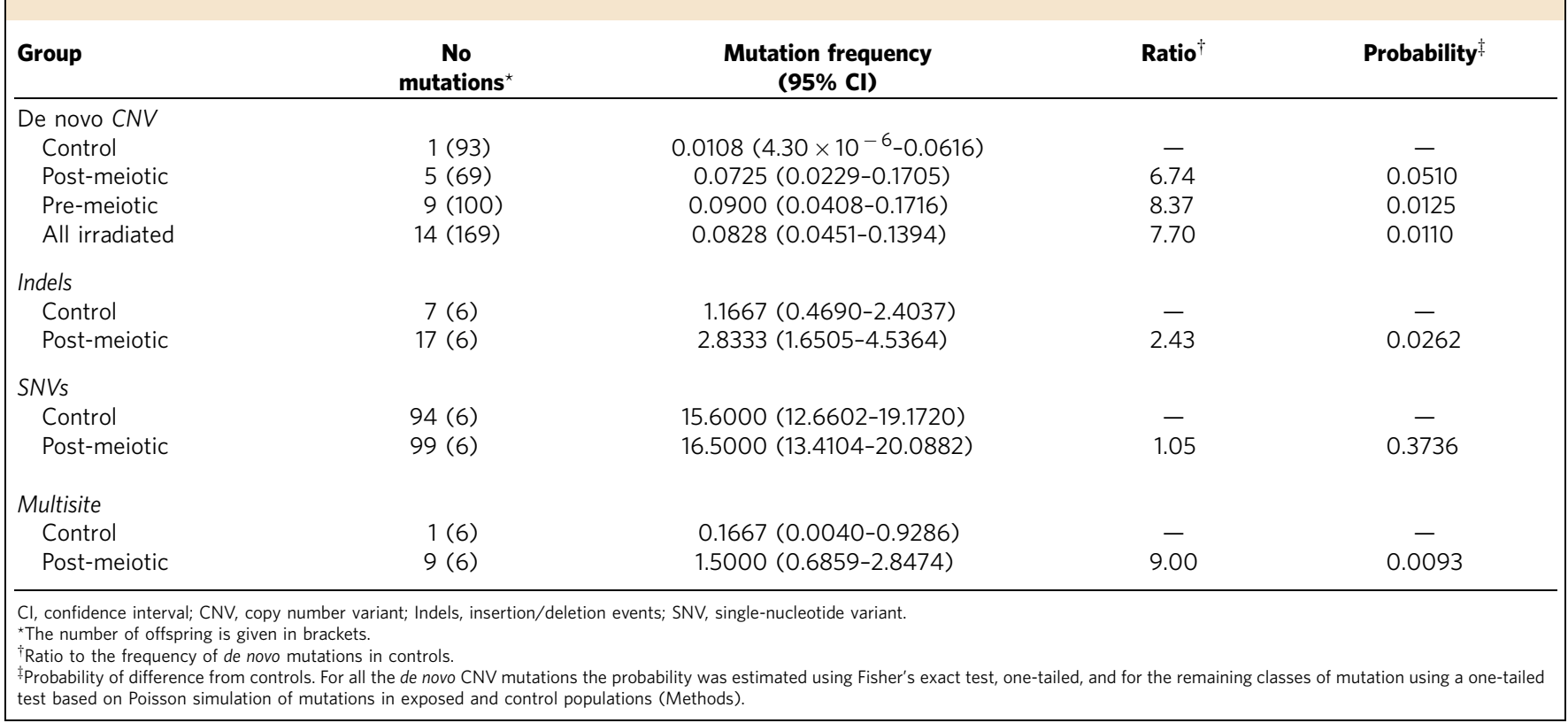

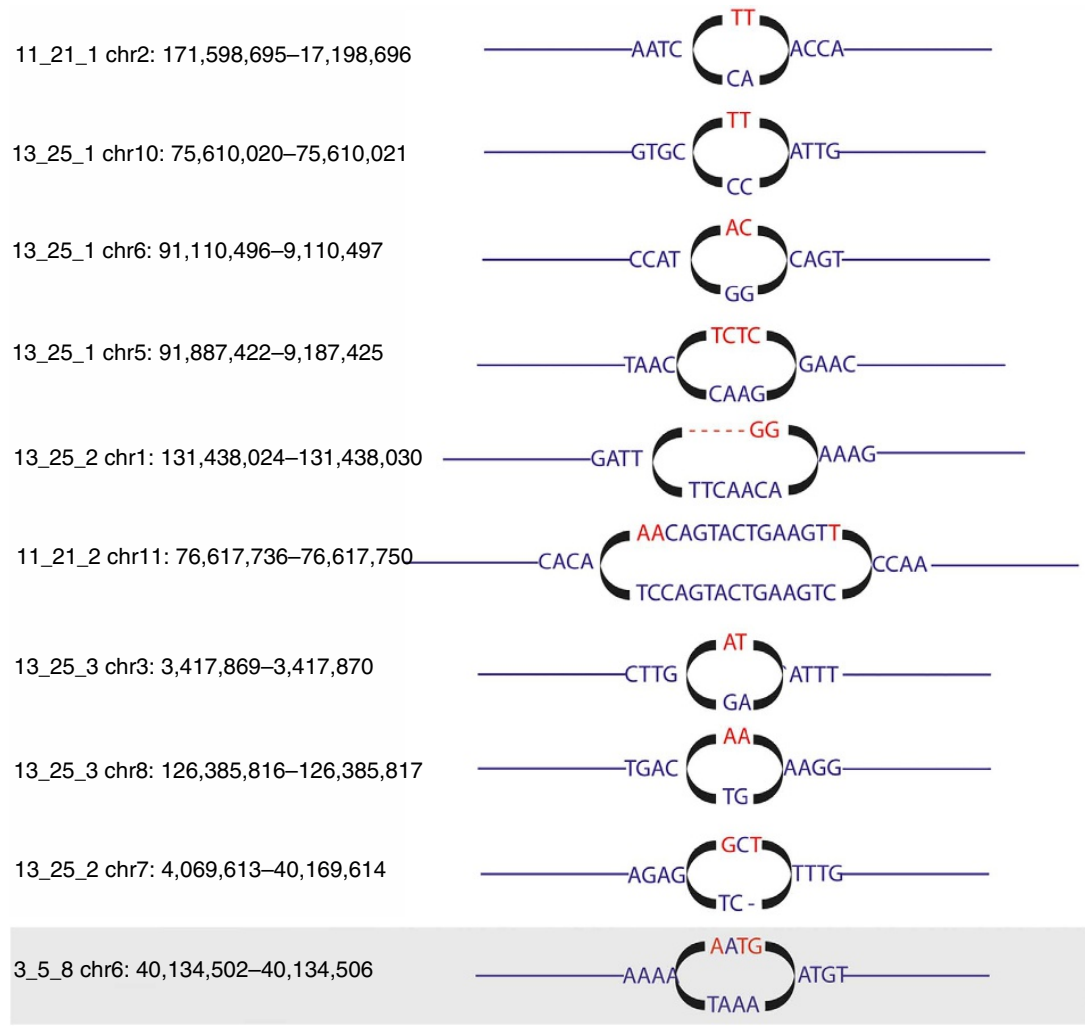

Figure 4 | All validated multisite de novo mutations found in this study. The reference sequence is shown in blue, mutant in red. Deletions are shown as dashed lines. The final site (boxed) was found in a control sample.

radiation-induced clustered damaged sites can lead to multiple nucleotide substitutions occurring within short stretches of DNA. It should be noted that as DNA repair in mature sperm is effectively shut down, all DNA damaged sites attributed to postmeiotic exposure are recognized and repaired after fertilization ${ }^{17}$. None of the spontaneous or induced de novo SNVs, indels or multisites are predicted to have had any phenotypic impact on the offspring; only two SNVs and two indels fall in exonic regions (in genes Adams20, Tmed9, Cep250 and H2afy), knockouts of which show no phenotypic changes.

\section{Conclusions}

In summary, we have validated the use of whole-genome approaches for characterizing the signatures of mutagen exposure in the mammalian germline and we report a systematic 
evaluation of the genome-wide effects of irradiation on mutation induction. Our in vivo genome-wide study differs from previous work, which has focused on either specific loci, or limited to bacterial cells and cell lines. We observed an increased magnitude of mutation induction with increasing size of the mutation event; paternal irradiation significantly increased the incidence of large ( $>5.5 \mathrm{~kb})$ CNVs, small (<50 bp) indels and multiple SNVs, whereas the total frequency of de novo SNV mutations among the offspring of exposed males was not significantly elevated. While we did not screen for intermediate sized $50 \mathrm{bp}$ to $5.5 \mathrm{~kb}$ structural variants, it is reasonable to expect this class of variation would also show significant mutation induction by IR given the shared mutational mechanisms with the larger (CNVs) and smaller (indel) mutation classes that are significantly induced. In our study, we did not observe any stratification in numbers or types of de novo mutation in the sex of the offspring, or any significant clustering of induced or spontaneous mutations in or between offspring (Fig. 5).

The genome-wide magnitude of mutation induction of SNVs that we observed may appear to be less than previously thought. It should be noted that the vast majority of radiation-induced
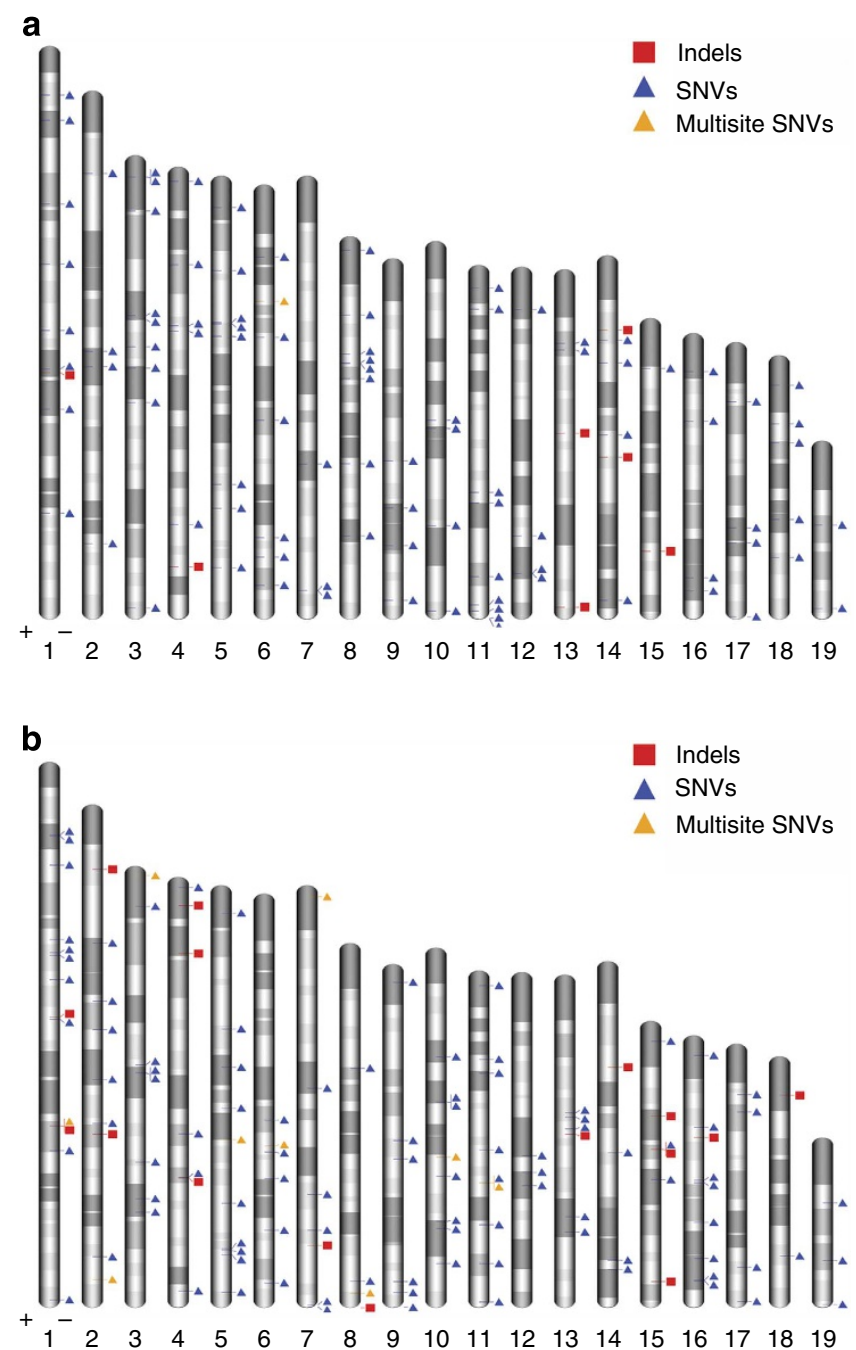

Figure 5 | Chromosomal distribution of de novo mutations found in this study. SNVs, CNVs, indels and multisite variants are shown for offspring that were whole-genome sequenced. The six control (a) and six exposed (b) offspring are shown on one plot, respectively. Plots drawn by Idiographica ${ }^{28}$
DNA damage is attributed to singly damaged bases ${ }^{1}$. According to the data from ref. 1, exposure to $3 \mathrm{~Gy}$ of acute X-rays should induce up to 6,000 damaged bases in the germline of irradiated males. Given that a diploid mammalian cell repairs 30,000 similar endogenous DNA lesions per day ${ }^{18}$, the induction of an extra 6,000 DNA lesions should not represent a sufficient challenge to the DNA repair machinery. In contrast, radiation-induced double-strand breaks and clustered damaged sites constitute the most dangerous types of DNA damage, as their repair is substantially delayed or compromised ${ }^{15,16}$. If not adequately repaired, these lesions would cause DNA rearrangements, including CNVs and indels, as well as clustered nucleotide substitutions, thus creating a specific signature of radiation exposure, described by our whole-genome study. We note that a signature of clustered mutations on exposure of Caenorhabditis elegans to chemical mutagens was recently described by Meier and colleagues $^{19}$. Applying the strategy adopted here to characterize germline mutation induction across a wide range of mutagenic exposures should lead to a step change in our understanding of the sensitivity of the mammalian germline to exogenous mutagens.

\section{Methods}

Mice. C57BL/6 (male) and CBA/Ca (female) mice were obtained from Harlan. Eight-week-old male mice were given whole-body acute irradiation of $3 \mathrm{~Gy}$ of $\mathrm{X}$-rays delivered at $0.5 \mathrm{~Gy} \mathrm{~min}^{-1}(250 \mathrm{kV}$ constant potential, HLV $1.5 \mathrm{~mm} \mathrm{Cu}$, Pantak industrial X-ray machine). Irradiated and sham-treated male mice were each mated with two age-matched non-irradiated CBA/Ca females either for 4 days immediately or 8 weeks post exposure. This design allows us to profile the effect of IR at two distinct stages of spermatogenesis. We referred to these stages as postmeiotic (mating immediately after irradiation) and pre-meiotic (mating after 8 weeks post-irradiation). Tissue samples were taken from all the parents and their 8 week-old offspring. The study protocol was approved by the University of Leicester Research Ethical Review Committee and performed under the Home Office project licence No. PPL 80/2267.

Microarray analysis. Genomic DNA was extracted from spleen and kidney by phenol-chloroform following standard protocols with $1 \%$ SDS and $300 \mu \mathrm{g} \mathrm{ml}^{-1}$ proteinase K (Roche Diagnostics Limited). DNA was quantified using ultraviolet spectroscopy (NanoDrop 1000, Thermo Scientific) and the quality checked on $1 \%$ agarose gel. For the comparative genomic hybridization, spleen DNA was sent to Roche NimbleGen Service Laboratory (Reykjavík, Iceland) for full service (sample labelling, hybridization and scanning). The labelled samples were hybridized to mouse ultra-high-resolution Comparative Genome Hybridization (CGH) arrays (Mouse 2.1M CGH, 080411_MM9_CGH_HX1, NimbleGen, Roche Diagnostics) with 2.1 million features and $1.1 \mathrm{~kb}$ median probe spacing. An equal molar dilution of father and mother genomic DNA was used as the reference genome for each offspring.

Quality control for hybridization data. The quality of hybridization data received from NimbleGen service centre was examined using two approaches. First, by generating a spatial plot for each of the array data and visually checking or any artefacts from the hybridization or inconsistencies between/ or within arrays. The plots were generated using Ringo package ${ }^{20}$ in $\mathrm{R}$ environment. From this QC measure, four of the 262 arrays failed owning to hybridization artefacts. The second approach employed median absolute deviation (MAD) of $\log 2$ ratios as the measure of noise in the data. The threshold for accepting array data was set at MAD $>0.16$ based on the distribution of the MAD values across the whole samples. Only array data that passed these QC procedures was used in calling CNVs.

Array based CNV discovery. The copy number calling was done using circular binary segmentation algorithm in R bioconductor DNAcopy package with additional post-quality control steps. Before the CNVs calling, the q-spline normalized data from NimbleGen was corrected for wave effect using an in-house algorithm. The threshold for accepting a CNV was set to comprise at least five probes with a median $\log 2$ ratio \pm 0.40 . Sex chromosomes were not included in the analysis.

Validation of CNVs. Real-time qPCR was used to validate the relative copy number of CNVs detected by array-CGH in two tissues: spleen and kidney. All qPCR experiments were performed on LightCycler 480 System (Roche Applied Science) using TaqMan detection chemistry. Mouse Transferrin receptor protein 1 (Tfrc1) was chosen as a reference target owning to its copy number consistency across our array-CGH data. Primers and probes were designed with Beacon 
Designer Free Edition (Premier Biosoft International) to accommodate duplexing TaqMan qPCR (primer and probe sequences provided in Supplementary Table 3). The reference probe was labelled with VIC dye at the $5^{\prime}$ end and minor groove binder quencher at the $3^{\prime}$ end. All the target probes were labelled with FAM dye and minor groove binder quencher at the $3^{\prime}$ end according to the manufacturer's protocol (Life Technologies). To ensure similar amplification efficiency between the target and the reference primers, standard curves were generated for each target and reference and only those in the range of 1.95-2.10 were accepted. Each qPCR assay was performed in quadruplicate and in duplex (a target and reference target Tfrc1). Each reaction contained $10 \mathrm{ng}$ of genomic DNA, $2 \times$ of SensiMix II probe (Bioline, UK), $0.1 \mu \mathrm{M}$ of target probe, $0.1 \mu \mathrm{M}$ of $T f r c 1$ probe, $0.5 \mu \mathrm{M}$ each of forward and reverse primers for the target and Trfrcl, respectively, in a total reaction volume of $5 \mu \mathrm{l}$. The $\mathrm{qPCR}$ thermal cycling conditions were as follows: initiation at $95^{\circ} \mathrm{C}$ for $10 \mathrm{~min}$ for hot start, followed by 45 cycles of $95^{\circ} \mathrm{C}$ for $10 \mathrm{~s}$ and $56^{\circ} \mathrm{C}$ for $1 \mathrm{~min}$. Data analysis was further performed using the $2^{-\Delta \Delta C_{T}}$ method $^{21}$.

Determining parental genotypes. The haplotype of parents and offspring with CNVs was determined by Sanger sequencing. Briefly, primers were designed to amplify a genomic region within the CNVs (deletions) by PCR. The PCR products were purified using the Zymoclean Gel DNA recovery kit (Zymo Research) and sequenced. The DNA sequences were aligned using Multiple Sequence Comparison by Log-Expectation ${ }^{22}$ to check for single-nucleotide polymorphisms (SNPs), which varied between the parents. The pattern of these SNPs was examined in the offspring with deleted CNV under consideration. Since we profiled first generation mice for CNVs, we expect to see heterozygotes for any given polymorphism, therefore loss of heterozyogsity enable us to trace the parental origin of the CNV.

Sequencing. DNA extracted from the spleen of parents and their offspring was pooled and sequenced using standard protocols and Illumina HiSeq technologies to an average depth of $25 \mathrm{X}$. The resultant sequence data were aligned to mouse reference NCBI37. Duplicate reads were removed, and BAM improvement carried out at lane and sample level according to GATK best practice ${ }^{23}$ before lane and sample data were merged. The total mapped sequence coverage was $23.3,24.6,30.4$, 24.1, 24.2, 25.5 reads per base for the offspring from irradiated fathers (11_21_1, 11_21_2, 11_21_3, 13_25_1, 13_25_2, 13_25_3), and 26, 26.4, 26.1, 23.1, 23.9, 29.2 reads per base for the control offspring $\left(2 \_3 \_1,2 \_3 \_2,2 \_3 \_3,3 \_5 \_1,3 \_5 \_4\right.$, $3 \_5 \_8$ ). The mapped reads per based for the parents were $23.4,24.4$ for the irradiated fathers (P11, P13), 25.8 and 24.1 for the mothers from the irradiated trios (PF21, PF25), and finally 30.6 and 22.5 mapped reads per base for the control mothers (PF3, PF5).

Variant calling. Variants were called using bcftools ${ }^{24}$ and samtools ${ }^{24}$, and standard settings. Variants were also called using samtools without BAQ alignment, although no additional candidates were added to the validation data set from this analysis.

De novo mutation calling. De novo mutations were called on the variants supplied by bcftools using DeNovoGear version 0.5 (ref. 25) and Triodenovo (http:// genome.sph.umich.edu/wiki/Triodenovo) using standard settings. DeNovoGear called 3,303, 3,284, 4,245, 3,500, 3,593 and 3,480 SNVs and small indel candidates in the autosomes of the offspring from irradiated fathers $\left(11 \_21 \_1,11 \_21 \_2\right.$, $11 \_21 \_3,13 \_25 \_1,13 \_25 \_2,13 \_25 \_3$, respectively) and 4,015, 4,334, 4,022, 3,172, 3,737 and 3,919 candidates in autosomes from the control offspring $\left(2 \_3 \_1,2 \_3 \_2\right.$, $233 \_3,351,354,355$ 8 respectively). Calls from the $\mathrm{X}$ chromosome were discarded as SNVs and indels showed a gender-associated inflation, which was not possible to correct for. DeNovoGear was re-run with a tenfold lower posterior probability threshold to capture low-quality indels and clustered SNVs.

Filtering. Candidate de novo variants were filtered to exclude simple sequence repeats and segmental duplications where we expected false positives to be enriched (on average $26-30 \%$ of candidates fall in a segmental duplication and $23-27 \%$ in a simple sequence repeat, although these categories are not exclusive of each other). Assuming a Poisson distribution for sequencing depth, sites with a depth greater than the 0.0001 quantile were removed due to the likelihood of mapping errors or low complexity repeats introducing false positives (generally $77-80 \%$ of candidate sites). Candidate sites where the de novo allele was present in either parent in greater than $0.05 \%$ of reads and where there were known SNPs in the parental strain were also removed on the grounds that they were likely to be inherited (4.06$6.21 \%$ and $0-4.73 \%$ of candidate sites, respectively). Once these filters were applied, $172,156,157,183,160$ and 149 indel and SNVs candidate de novos remained for validation in the offspring from irradiated fathers $\left(11 \_21 \_1,11 \_21 \_2,11 \_21 \_3\right.$, $1325 \_1,13 \_25 \_2$ and $13 \_25 \_3$ ), along with $174,183,168,166,172$ and 149 small indel and SNVs candidate de novos for control offspring $\left(2 \_3 \_1,2 \_3 \_2,2 \_3 \_3\right.$, 3_5_1, 3_5_4, 3_5_8).

Given the potential diverse nature of the mutations that may be induced in the offspring from irradiated fathers, we tried three different strategies to increase the numbers of candidate variants before validation. First, we tried a different de novo variant caller (Triodenovo) to call de novo mutations in all trios, using our standard variant calls. Visual inspection showed that most high-quality candidate calls were called by both callers. However, 1,171 sites that were called by Triodenovo and not by DeNovoGear across all 12 trios were manually reviewed, leading to 46 plausible de novo candidates. After filtering these 46 Triodenovo calls using the same criteria as above, 13 candidates remained that were added to the validation callset (four calls in $2 \_3 \_1$, three calls in 11_21_1, three calls in 3_5_1 and one call each in 11_21_3, 13_35_3 and 3_5_8). Second, we re-ran DeNovoGear using standard variant calls but decreased the posterior probability threshold by a factor of 10 to allow for more low-quality indel de novo calls. After filtering as above, 811 sites were manually reviewed and 46 were added to the callset for validation. $(5,4,3,5,4$ and 3 sites from offspring from irradiated fathers and 5, 2, 6, 4, 3, 3 from offspring from control fathers, respectively). Last, we re-ran Samtools without BAQ realignment in order to increase the number of clustered mutations called by Samtools. Candidate sites were filtered as before and any previously considered candidates were removed. This led to $120-200$ additional candidates' sites per trio where there were two candidate sites directly adjacent to each other. A proportion of these sites were manually reviewed but no further candidates were added to the validation callset. In total, 2,048 candidate de novo mutations were put forward for validation (Supplementary Table 4). We intentionally included many lowconfidence calls in our validation callset in order to maximize sensitivity.

Experimental validation. A sequence of $400 \mathrm{bp}$ flanking the putative mutation were extracted, and primers were designed using Primer 3 (ref. 26) so that the mutation lay in the centre of a 300- to 425-bp PCR product. Primers were designed to have a GC clamp, be optimally $20 \mathrm{bp}$ in length and with a melting temperature of $60^{\circ}$. All primers were ordered from Sigma Genosys. Using this strategy, 2,034/ 2,048 regions resulted in successful primer design. The region-specific primers were then tailed with a standard forward and reverse sequencing primer.

Forward sequencing primer $5^{\prime}$-ACACTCTTTCCCTACACGACGCTCTTCC GATCT- $3^{\prime}$

Reverse sequencing primer $5^{\prime}$-CGGTCTCGGCATTCCTGCTGAACCGCTCT TCCGATCT- $3^{\prime}$

These primers were used to generate PCR products using RedAccuLATaq from Sigma Aldrich, $10 \mu \mathrm{M}$ primer and a touchdown PCR protocol. Each sample was then tagged with Illumina primers and a primer specific to each individual in a second round of PCR, using biotyinlated primers, Kapa HiFi Taq (Kapa Biosystems) Taq and $5 \mu \mathrm{l}$ of first round PCR product as template.

The second round PCR products were pooled by individual and then cleaned up using Agencourt Ampure SPRI beads from Beckman Coulter. All individuals were then pooled together, and the pool balances checked. Three lanes of the balanced pools were run on an Illumina Miseq platform ( $250 \mathrm{bp}$ paired end reads), leading to an average of $100 \mathrm{X}$ coverage across the candidate site of interest. The resultant sequence data were merged by individual and annotated with read counts at the candidate site using an in house python script. An in house R script (http://www.Rproject.org) was then used to allocate a likelihood to each candidate variant being a true de novo mutation, an inherited variant or a false positive call, based on the allele counts of the parents and child at that locus. A proportion of the SNV candidates as well as all of the indel candidates were reviewed manually using Integrative Genomics Viewer (IGV). A summary of validation is given in Supplementary Table 5.

Haplotyping of de novo SNVs and indels. We used the read-pair algorithm supplied with the DeNovogear software to determine the parent of origin of our validated de novo mutations using the deep whole-genome sequence data. DeNovoGear uses information from flanking variants that are not shared between parents to calculate the haplotype on which the mutation arose. Using this technique, we were able to confidently assign the parental haplotype in 49/224 validated de novo mutations.

Mutation rate estimation. We calculated a mutation rate for autosomal SNVs in each individual as follows: first, we calculated the proportion of the genome not covered in our analysis because of the depth of the whole-genome sequencing: Bedtools ${ }^{27}$ was used to calculate the proportion of the genome not considered in our analysis due to low- or high-sequence depths for each individual (mean 8.4\%) We then calculated what proportion of sites were removed by our whole-genome filters (simple sequence repeats and segmental duplications) after the depth filters were applied (average $2.7 \%$ ). Last, we used the posterior probability supplied by DeNovoGear to calculate what proportion of sites that were not validated (failed validation or removed by to filters), would be likely to be true de novo mutations

Calculation of rate significance. We assessed the significance of the difference in numbers of SNV, multisite and indel mutations between offspring of exposed and control fathers using a one-tailed test based on Poisson simulation of the null distribution of the difference in the numbers of mutations observed in offspring of exposed and control fathers, assuming the same mutation rate across exposed and control offspring based on the average mutation rate across the two groups. Significance was estimated by calculating the proportion of the 100,000 simulations that gave an excess of numbers of mutations in case offspring compared with control offspring that was the same or greater than observed (Supplementary Fig. 1). 
Functional annotation of validated de novo variants. Annotation of SNVs, indels and multisite variants were carried out using ANNOVAR (ref. 28).

\section{References}

1. Frankenberg-Schwager, M. Induction, repair and biological relevance of radiation-induced DNA lesions in eukaryotic cells. Radiat. Environ. Biophys. 29, 273-292 (1990).

2. UNSCEAR. Hereditary Effects of Radiation (United Nations, , 2001).

3. Nakamura, N. et al. Radiation effects on human heredity. Ann. Rev. Genet 47, 51-68 (2013).

4. Searle, A. G. Mutation induction in mice. Adv. Radiat. Biol 4, 131-207 (1974).

5. Russell, W. L. \& Kelly, E. M. Mutation frequencies in male mice and the estimation of genetic hazard of radiation in men. Proc. Natl Acad. Sci. USA 79, 525-544 (1982).

6. Egan, C. M., Sridhar, S., Wigler, M. \& Hall, I. R. Recurrent DNA copy number variation in the laboratory mouse. Nat. Genet. 39, 1384-1389 (2007).

7. Campbell, I. M. et al. Parental somatic mosaicism is underrecognized and influences recurrence risk of genomic disorders. Am. J. Hum. Genet. 95, 173-182 (2014).

8. Dubrova, Y. E. et al. Stage specificity, dose response, and doubling dose for mouse minisatellite germ-line mutation induced by acute radiation. Proc. Natl Acad. Sci. USA 95, 6251-6255 (1998).

9. Campbell, C. D. \& Eichler, E. E. Properties and rates of germline mutations in humans. Trends Genet. 29, 575-584 (2013).

10. The Genome of the Netherlands Consortium. Whole-genome sequence variation, population structure and demographic history of the Dutch population. Nature Genet. 46, 818-825 (2014).

11. Wade, C. M. et al. The mosaic structure of variation in the laboratory mouse genome. Nature 420, 574-577 (2002).

12. Schrider, D. R. et al. Pervasive multinucleotide mutational events in eukaryotes. Curr. Biol 21, 1051-1054 (2011)

13. Terekhanova, N. V. et al. Prevalence of multinucleotide replacements in evolution of primates and Drosophila. Mol. Biol. Evol. 30, 1315-1325 (2013).

14. Ward, J. F. in: DNA Damage and Repair vol. 2Nickoloff, J. A. \& Hoekstra, M. F. (edspp 65-84 (Human, 1998).

15. Georgakis, A. G., O’Neill, P. \& Stewart, R. Induction and repair of clustered DNA lesions: What do we know so far? Radiat. Res. 180, 100-109 (2013).

16. Eccles, L. J., O’Neill, P. \& Lomax, M. E. Delayed repair of radiation induced clustered damage: Friend or foe? Mutat. Res. 711, 134-141 (2011).

17. Baarends, W. M., van der Laan, R. \& Grootegoed, A. J. DNA repair and gametogenesis. Reproduction 121, 31-39 (2001).

18. Lindahl, T. \& Anderson, B. Repair of endogenous DNA damage. Cold Spring Harbor Symp. Quant. Biol 65, 127-133 (2000).

19. Meier, B. et al. C. elegans whole-genome sequencing reveals mutational signatures related to carcinogens and DNA repair deficiency. Genome Res. 24, 1624-1636 (2014)

20. Toedling, J. et al. Ringo - an R/Bioconductor package for analyzing ChIP-chip readouts. BMC Bioinformatics 8, 221 (2007)

21. Livak, K. J. \& Schmittgen, T. D. Analysis of relative gene expression data using real-time quantitative PCR and the $2^{-\Delta \Delta}$ CT method. Methods 25, 402-408 (2001).

22. Edgar, R. C. MUSCLE: multiple sequence alignment with high accuracy and high throughput. Nucleic Acids Res. 32, 1792-1797 (2004).
23. Van der Auwera, G. A. et al. From FastQ data to high-confidence variant calls: The genome analysis toolkit best practices pipeline. Curr. Protoc. Bioinform. 43 11.10.1-11.10.33 (2013).

24. Li, H. et al. The Sequence alignment/map (SAM) format and SAMtools. Bioinformatics 25, 2078-2079 (2009).

25. Ramu, A. et al. DeNovoGear: de novo indel and point mutation discovery and phasing. Nat. Methods 10, 985-987 (2013).

26. Untergasser, A. et al. Primer3--new capabilities and interfaces. Nucleic Acids Res. 40, e115 (2012).

27. Quinlan, A. R. \& Hall, I. M. BEDTools: a flexible suite of utilities for comparing genomic features. Bioinformatics 26, 841-842 (2010).

28. Wang, K., Li, M. \& Hakonarson, H. ANNOVAR: functional annotation of genetic variants from high-throughput sequencing data. Nucleic Acids Res. 38, e164 (2010).

\section{Acknowledgements}

We thank the Division of Biomedical Services, University of Leicester for their expert animal care and Tomas Fitzgerald for his help with CNV calling. We thank the Wellcome Trust for their support (WT091106 and WT098051).

\section{Author contributions}

Y.E.D. and M.E.H. supervised the project, generated the original hypothesis and designed the study. A.B.A and S.J.L. performed the experiments. A.B.A, S.J.L., Y.E.D. and M.E.H. analysed the data and wrote the manuscript. All authors read and approved the final manuscript.

\section{Additional information}

Accession codes: The microarray data were deposited in the Gene Expression Omnibus (GEO), submission number GSE65521; the whole-genome sequences were deposited in the Sequence Read Archive under Study number ERP001221, with accession numbers ERX111346, ERX111347, ERX111348, ERX111349, ERX111350, ERX111351, ERX111352, ERX111353, ERX111354, ERX111355, ERX111356, ERX111357, ERX111358, ERX111359, ERX111360, ERX111361, ERX111362, ERX111363, ERX111364, ERX111365, ERX111366 and ERX111367.

Supplementary Information accompanies this paper at http://www.nature.com/ naturecommunications

Competing financial interests: The authors declare no competing financial interests.

Reprints and permission information is available online at http://npg.nature.com/ reprintsandpermissions/

How to cite this article: Adewoye, A. B. et al. The genome-wide effects of ionizing radiation on mutation induction in the mammalian germline. Nat. Commun. 6:6684 doi: $10.1038 /$ ncomms7684 (2015)

(c) (i) This work is licensed under a Creative Commons Attribution 4.0 International License. The images or other third party material in this article are included in the article's Creative Commons license, unless indicated otherwise in the credit line; if the material is not included under the Creative Commons license, users will need to obtain permission from the license holder to reproduce the material. To view a copy of this license, visit http://creativecommons.org/licenses/by/4.0/ 